\title{
Microcarrier-Assisted Inorganic Shelling of Lead Halide Perovskite Nanocrystals
}

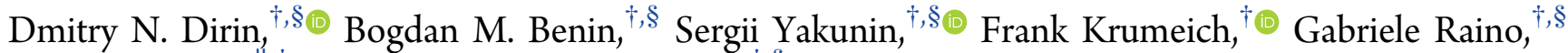
Ruggero Frison, ${ }^{\|, \ddagger}$ and Maksym V. Kovalenko* $*, \S[0$

${ }^{\dagger}$ Institute of Inorganic Chemistry, Department of Chemistry and Applied Biosciences, ETH Zürich, CH-8093 Zürich, Switzerland ${ }^{\S}$ Laboratory for Thin Films and Photovoltaics, Empa - Swiss Federal Laboratories for Materials Science and Technology, CH-8600 Dübendorf, Switzerland

"Department of Chemistry, University of Zürich, CH-8057 Zürich, Switzerland

${ }^{\ddagger}$ Center for X-ray Analytics, Empa - Swiss Federal Laboratories for Materials Science and Technology, CH-8600 Dübendorf, Switzerland

\section{Supporting Information}

ABSTRACT: The conventional strategy of synthetic colloidal chemistry for bright and stable quantum dots has been the production of epitaxially matched core/shell heterostructures to mitigate the presence of deep trap states. This mindset has been shown to be incompatible with lead halide perovskite nanocrystals (LHP NCs) due to their dynamic surface and low melting point. Nevertheless, enhancements to their chemical stability are still in great demand for the deployment of LHP NCs in light-emitting devices. Rather than contend with their attributes, we propose a method in which we can utilize their dynamic, ionic lattice and

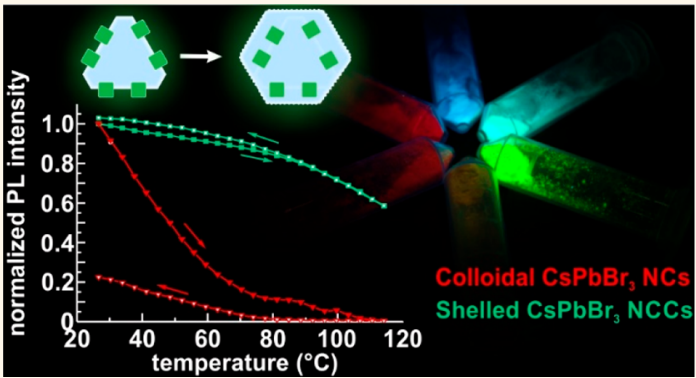
uniquely defect-tolerant band structure to prepare non-epitaxial saltshelled heterostructures that are able to stabilize these materials against their environment, while maintaining their excellent optical properties and increasing scattering to improve out-coupling efficiency. To do so, anchored LHP NCs are first synthesized through the heterogeneous nucleation of LHPs onto the surface of microcrystalline carriers, such as alkali halides. This first step stabilizes the LHP NCs against further merging, and this allows them to be coated with an additional inorganic shell through the surface-mediated reaction of amphiphilic $\mathrm{Na}$ and Br precursors in apolar media. These inorganically shelled NC@carrier composites offer significantly improved chemical stability toward polar organic solvents, such as $\gamma$-butyrolactone, acetonitrile, $N$-methylpyrrolidone, and trimethylamine, demonstrate high thermal stability with photoluminescence intensity reversibly dropping by no more than $40 \%$ at temperatures up to $120{ }^{\circ} \mathrm{C}$, and improve compatibility with various UV-curable resins. This mindset for LHP NCs creates opportunities for their successful integration into next-generation light-emitting devices.

KEYWORDS: lead halide perovskite, luminescence, nanocrystals, stability, core/shell

olloidal lead halide perovskite nanocrystals (LHP NCs) have recently emerged as a novel class of luminescent materials with outstanding optical properties, such as high photoluminescence quantum yields (PL $\mathrm{QY}=50-90 \%),{ }^{1-5}$ pure PL colors tunable across the entire visible range, ${ }^{1}$ PL lifetimes varying from a few to a few hundreds of nanoseconds depending on composition and morphology, ${ }^{6}$ suppressed blinking, ${ }^{7}$ optical properties that are tolerant to intrinsic defects, ${ }^{8}$ superfluorescence from superlattices, ${ }^{9}$ and an unusual electronic fine structure as evidenced from single-dot studies. ${ }^{10,11}$ Following the first reports on ligand-capped colloidal formamidinum LHPs $\left(\mathrm{FAPbX}_{3}, \mathrm{X}=\right.$ $\mathrm{Cl}, \mathrm{Br}, \mathrm{I})^{12-15}$ and the fully inorganic $\mathrm{CsPbX}_{3} \mathrm{NCs}{ }^{1}$ considerable attention has been devoted to various aspects of their chemistry: post-synthetic anion exchange, ${ }^{16-18}$ shape- engineering, ${ }^{6,19-23}$ self-assembly, ${ }^{9,24}$ synthesis exploration, ${ }^{6,25-28}$ and the search for alternative perovskite-like compositions. $^{29-39}$

From a practical viewpoint, the most anticipated near-term application of LHP NCs is as green phosphors in backlit films for LCD displays. In this regard, and somewhat contrary to general belief, LHP NCs are RoHS-compliant, unlike CdSebased QDs, because their strong optical absorption allows the lead concentration in the emitting layer to be kept well below $1000 \mathrm{ppm}$, which is the allowed concentration for any

Received: July 12, 2019

Accepted: October 4, 2019

Published: October 4, 2019 
Scheme 1. Encapsulation of LHP NCs into Inorganic Microcrystals ${ }^{a}$

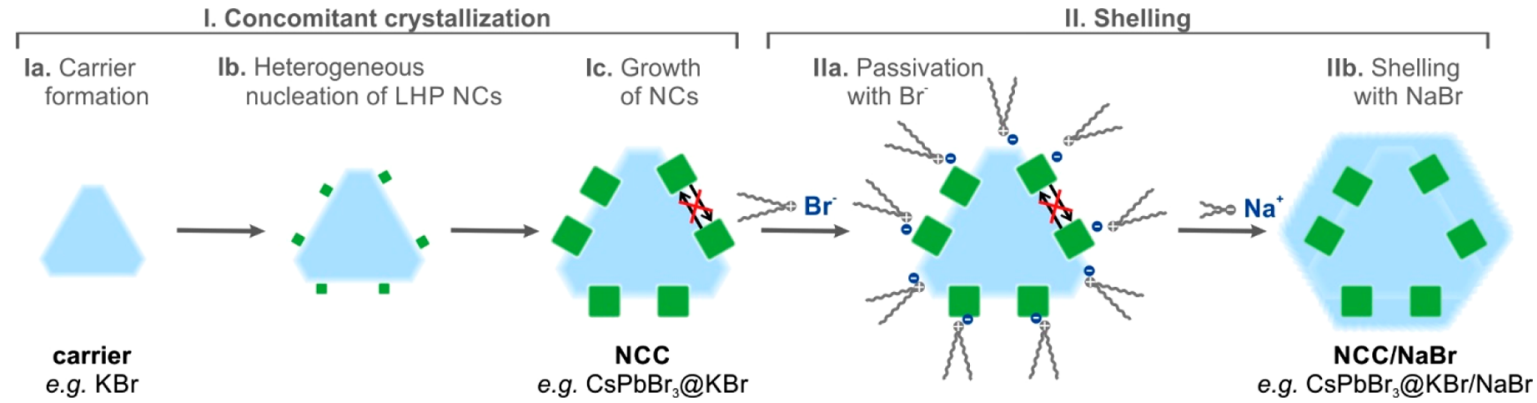

${ }^{a}$ In the first step $(\mathrm{Ia}-\mathrm{c})$, LHP NCs heterogeneously nucleate and grow on the surface of the microcrystalline carriers $\left(\mathrm{NaX}, \mathrm{KX}, \mathrm{RbX} \mathrm{Cs} \mathrm{PbX}_{6}\right.$, $\left(\mathrm{Cs}_{x} \mathrm{Rb}_{1-x}\right)_{4} \mathrm{PbX}_{6}, \mathrm{MgX}_{2}, \mathrm{CaX}_{2}, \mathrm{SrX}_{2}, \mathrm{BaX}_{2}$, and $\mathrm{ZnX}_{2}$ where $\mathrm{X}=\mathrm{Cl}, \mathrm{Br}$, I or their mixtures.) In Ic, the crossed-arrows indicate that inter-NC merging is strongly diminished. The obtained NCCs are then shelled with $\mathrm{NaBr}$ in the second step of the synthesis through the direct reaction of amphiphilic $\mathrm{Na}$ and $\mathrm{Br}$ precursors in an apolar solvent (IIa,b). The entire process yields the final, shelled polycrystalline $\mathrm{NCC} / \mathrm{NaBr}$ particles.

separable part of lead-containing devices. This RoHS regulation is independent of the encapsulation strategy and any potential leakage. ${ }^{40,41}$ At the same time, green-emitting LHP NCs offer superior optical properties compared to the currently commercialized benchmark InP-based QDs with PL QYs of about $85-90 \%$ (against 60\% for $\mathrm{InP}$ ) and narrow emission line widths of $20 \mathrm{~nm}$ (compared to $40 \mathrm{~nm}$ for InP). ${ }^{41}$ Furthermore, some estimates indicate that the superior absorption of blue light by LHP NCs over InP QDs may be sufficient to replace color filters (which can absorb up to 2/3 of emitted light in LCDs) with pixellated arrays of NCs. ${ }^{40,42-44}$ This can be a step forward toward more energy-efficient $\mathrm{QD}$ LCDs. ${ }^{45}$

In this perspective, strong efforts have been dedicated toward improving the stability of colloidal LHP NCs. ${ }^{46-73}$ Still, colloidal LHP NCs struggle from several stability issues that hamper their deployment in applications such as LCD displays. $^{47,74,75}$ First of all, ionic LHPs are moderately to highly soluble in polar solvents, which limits the processing after colloidal synthesis. Second, the highly dynamic binding of native ligands allows access of any damaging species to the surface of NCs. ${ }^{28}$ Third, the relatively low melting point of LHPs assists the sintering of NCs when they are in close proximity. All of these instabilities promote NC ripening, the rapid and uncontrolled evolution of morphology and size as well as coalescence and sintering, which can be accelerated by oxygen, moisture, and light. ${ }^{74}$ This hampers the post-synthetic processing of LHP NCs, especially in the presence of polar molecules and at elevated temperatures. Most of these issues can be overcome by properly isolating individual NCs from each other and from their environment. There are several common ways to accomplish this: embedding colloidal LHP NCs in a polymer film, ${ }^{50,52,53}$ in glasses, ${ }^{55,56,58}$ in lipid micelles, ${ }^{54}$ or in $\mathrm{Pb}$-containing salts, ${ }^{53,68,71,76,77}$ coprecipitation of colloidal LHP NCs with salts, ${ }^{59,78}$ modifying their surface, ${ }^{65-70,79-81}$ or capping the NCs with a shell. ${ }^{47,51,62-64,73}$ These approaches are well-established for conventional and structurally more rigid semiconductor NCs, typically made of II-VI, III-V, and IV-VI compounds. However, most of these known protocols require the treatment of NCs with polar solvents or molecules which, in the case of LHPs, accelerate the ripening of NCs. ${ }^{12,51}$ Recently, we and several other groups have proposed a synthetic approach which overcomes this problem by isolating NCs from each other already during the nucleation step. ${ }^{8,82-85}$ Specifically, LHP NCs were synthesized inside a chemically robust mesoporous matrix that prevented NCs from ripening and retarded the dissolution of LHPs in the presence of polar moieties.

These previous works have revealed several specific attributes of LHP NCs that suggest re-evaluating their engineering. First, as a result of their defect-tolerance, LHP NCs do not require passivation for bright PL as they are rather insensitive to dangling bonds. In addition to this, their highly dynamic lattice allows their surface to rearrange to adhere to a variety of materials: $\mathrm{SiO}_{2}, \mathrm{Al}_{2} \mathrm{O}_{3}, \mathrm{TiO}_{2}, \mathrm{Cs}_{4} \mathrm{PbBr}_{6}$, etc. Finally, LHP NCs are the only currently known semiconductor material to exhibit bright emission with PL QY approaching unity in the weak-confinement regime. This allows them to be synthesized with high polydispersity and large sizes, while still retaining exceptionally narrow emission line widths down to 18 $\mathrm{nm}^{72}$

Additionally, these findings suggest that there is no need to stick to the concept of thin, epitaxial shelling of individual QDs when using LHP NCs. In contrast to conventional QDs, where the complete and uniform epitaxial shelling of well-isolated colloidal particles is required, LHP NCs can retain their excellent optical properties even when directly embedded into non-epitaxial micrometer-scale composites or templates. ${ }^{8,82-84}$ The use of such micron-sized composites allows us to address a critical issue in QD-based backlit films for LCDs - poor light out-coupling efficiency. ${ }^{86}$ This issue is related to the waveguiding of light within the emissive layer, which has two primary loss mechanisms. First, it reduces the fraction of light emitted from the frontal plane of the device toward the liquid crystal layer. Second, the wave-guiding increases the effective optical path and thereby increases the likelihood of reabsorption by other QDs. These losses can be particularly high in QD-based down-converting layers as a result of their generally small Stokes shifts, leading to increased overlaps between absorption and emission bands. ${ }^{87}$ A common strategy toward reducing these losses involves the incorporation of highly scattering microparticles (e.g., $\mathrm{SiO}_{2}, \mathrm{ZnO}, \mathrm{BaSO}_{4}, \mathrm{ZrO}_{2}$ ) into the down-converting layer to improve the light outcoupling efficiency. ${ }^{88,89}$

Herein we propose a facile, non-colloidal synthetic route that accomplishes both aforementioned goals - creating bright LHP emitters along with photonic engineering for lightoutcoupling. Specifically, luminescent $\mathrm{APbX}_{3} \mathrm{NCs}(\mathrm{A}=\mathrm{Cs}$ or $\mathrm{FA}$; $\mathrm{X}=\mathrm{Cl}, \mathrm{Br}, \mathrm{I}$ ) were heterogeneously nucleated onto the surface of inorganic microcrystalline carriers (nanocrystals on carriers; NCCs). We show that the anchoring of LHP NCs significantly stabilizes them against merging and allows them to 


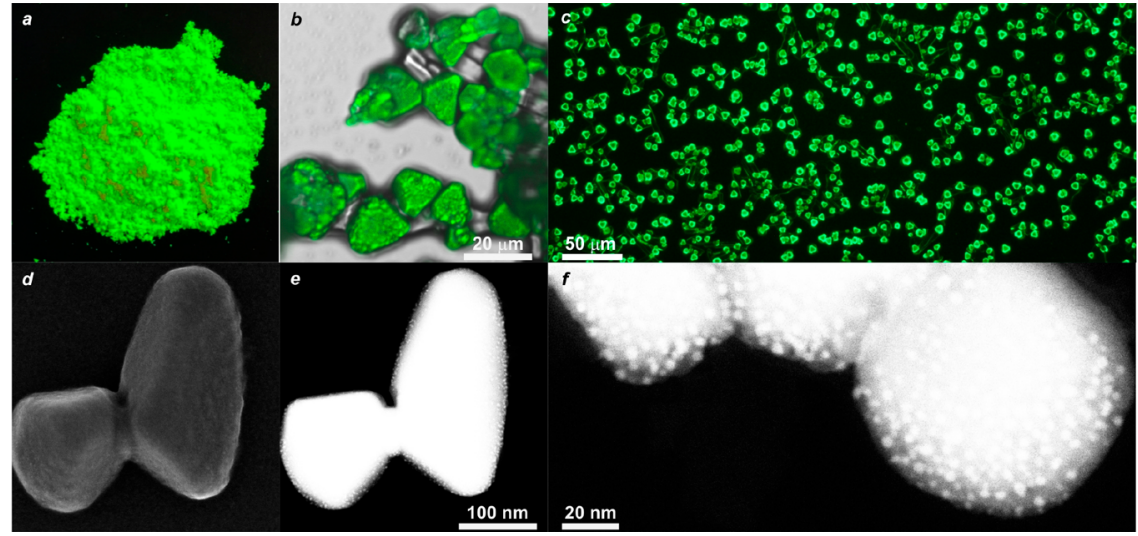

Figure 1. (a) Visual appearance of $\mathrm{FAPbBr}_{3} @ \mathrm{NaBr} \mathrm{NCC}$ powder under UV light and (b) a confocal microscopy image. (c) FAPbBr 3 @NaBr NCC powder under UV light with an optical microscope. STEM images of $\mathrm{CsPbBr}_{3} @ \mathrm{RbBr}$ NCC powder in (d) secondary electron mode and (e) HAADF mode. (f) A zoomed-in view of several NCCs in HAADF-mode.

be shelled with transparent and chemically robust $\mathrm{NaBr}$ salt matrices. The final shelled $\mathrm{NCC} / \mathrm{NaBr}$ exhibit improved stability against a vast variety of solvents and can be used in processes that are otherwise inaccessible or very harmful for colloidal LHP NCs, such as encapsulation into industrially important acrylate-based polymers including silicone urethane acrylate, hydroxyethyl methacrylate, and poly(vinyl acetate). ${ }^{90,91}$ Furthermore, the presence of highly scattering, polydisperse microcrystals with sizes in the range of $0.1-1 \mu \mathrm{m}$ hinders wave-guiding and improves the efficiency of light outcoupling, making these composite materials well-suited for use in LCD displays. ${ }^{88,89}$

\section{RESULTS AND DISCUSSION}

The synthesis begins with the concomitant crystallization of an inorganic salt, hereafter called a carrier, and LHP NCs from a single solution in a polar solvent. Crystallization can be induced either by evaporating the solvent or by the addition of a suitable antisolvent. The concentrations and solvents are chosen in such a way that the carrier microcrystals start to precipitate first and enable the heterogeneous nucleation of LHP NCs, which is followed by their growth (Scheme 1). Through this process, the LHP NCs are anchored to the surface of the carrier (NCC) and are thereby stabilized against merging. This also allows for further processing in the presence of polar molecules. In the next step, a shell of an alkali halide or another inorganic salt can be grown onto these composite materials, thus completing the encapsulation of the LHP NCs (Scheme 1). The proposed approach has been tested for the encapsulation of Cs- and FA-based LHP NCs into NaX, KX, $\mathrm{RbX}, \mathrm{Cs}_{4} \mathrm{PbX}_{6},\left(\mathrm{Cs}_{x} \mathrm{Rb}_{1-x}\right)_{4} \mathrm{PbX}_{6}, \mathrm{MgX}_{2}, \mathrm{CaX}_{2}, \mathrm{SrX}_{2}, \mathrm{BaX}_{2}$, and $\mathrm{ZnX}_{2}$ matrices where $\mathrm{X}=\mathrm{Cl}, \mathrm{Br}$, I or their mixtures. We selected these carriers due to their transparency to visible light and their suitable solubility in polar aprotic organic solvents, which is typically a few times lower than the solubility of LHPs. Some of these carriers $\left(\mathrm{MgBr}_{2}, \mathrm{CaBr}_{2}, \mathrm{ZnBr}_{2}\right)$ were too hygroscopic and therefore led to LHP NCs with poor stability. From all the other carriers, each carrier-LHP pair required the molar ratio to be adjusted due to the difference in carriers' solubilities, as discussed below.

To prepare these materials, solutions of an alkali-halide $\left(\mathrm{NaBr}, \mathrm{KBr}\right.$, or $\mathrm{RbBr}$ ), $\mathrm{CsBr}$ or $\mathrm{FABr}$, and $\mathrm{PbBr}_{2}$ in dimethyl sulfoxide with a small addition of $\mathrm{N}$-methylformamide are correspondingly mixed in a desired molar ratio (see SI and
Table S1 for details). This mixture was then drop-cast onto a glass substrate and typically dried at $70{ }^{\circ} \mathrm{C}$ with $25-50 \%$ relative humidity $(\mathrm{RH})$ for $3 \mathrm{~h}$ and then allowed to cool to room temperature. The synthesis produces a homogeneous, microcrystalline powder with morphologies and optical properties that are very different from either of the precursors (Figures 1a and S1).

Confocal and optical microscopy shows roughly faceted microcrystals that often exhibit a tetrahedral habit, which is typical for alkali halides (Figure $1 \mathrm{~b}, \mathrm{c}$ ). These microcrystals are only slightly colored but yield bright green luminescence under UV-excitation. This combination of crystal faceting and bright emission implies that the emission centers are mainly localized on the surface of the microcrystals and that they are not, or at least not always, embedded inside. Confocal microscopy confirms that LHP NCs are primarily localized on the surface of microcrystals (Figure S2). The high polydispersity is favorable for these microcrystals, as it can significantly improve the total scattering within the system, resulting in improved out-coupling efficiencies if introduced as down-converting layers in LCDs.

Scanning transmission electron microscopy in secondary electron (SE) and high-angle annular dark-field (HAADFSTEM) modes confirm the presence of heavy-atom nanocrystalline inclusions on or near the surface of the microcrystals (Figures $1 \mathrm{~d}-\mathrm{f}$ and $\mathrm{S} 3$ ). We note that electron microscopy of the interior of microcrystals is not feasible due to their large thickness. Furthermore, electron microscopy of such composite materials is generally difficult due to the high volatility of metal halides under an electron beam. ${ }^{92}$ However, STEM qualitatively shows the high concentration of LHP NCs and their small size $(c a .5-10 \mathrm{~nm})$, which is in agreement with a PL peak position of $510 \mathrm{~nm}$ for this sample $\left(\mathrm{RbBr}: \mathrm{CsPbBr}_{3}\right.$ 10:1) and corresponds to that of colloidal 8-10 nm NCs. ${ }^{1,43}$ Energy-dispersive $\mathrm{X}$-ray spectroscopy shows the presence of $\mathrm{Cs}, \mathrm{Rb}, \mathrm{Pb}$, and $\mathrm{Br}$, which together with the HAADF-STEM images imply that the carrier consists of alkali bromide, whereas bright $\mathrm{NCs}$ are Pb-based (Figure S3). Furthermore, powder X-ray diffraction (XRD) confirms the presence of a crystalline carrier (alkali halides or $\mathrm{Cs}_{4} \mathrm{PbBr}_{6}$ ) as the dominant phase (Figure S4). However, XRD measured on a conventional powder diffractometer is not able to reveal the presence of $\mathrm{CsPbBr}{ }_{3} \mathrm{NCs}$ due to the significant, size-related broadening of relevant reflections and the small amount of LHP compared to 

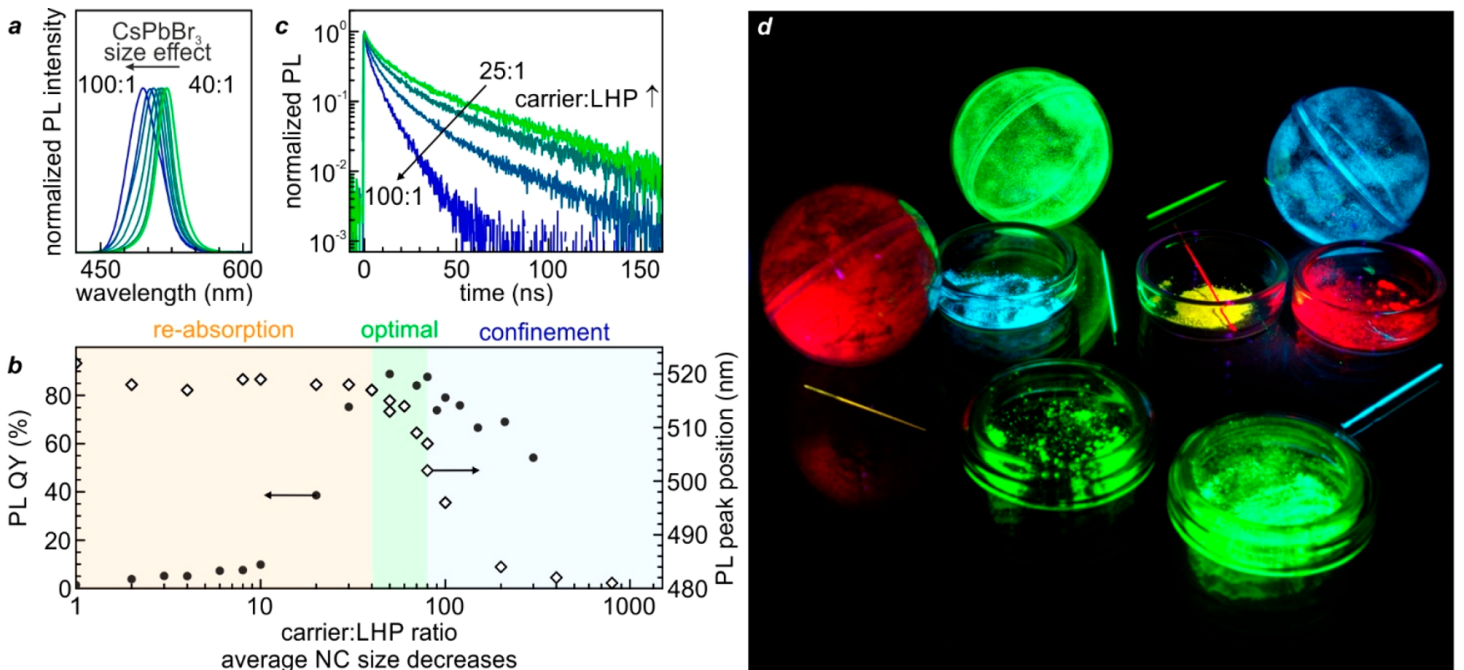

Figure 2. Optical properties of unshelled CsPbX NCCs. (a) PL tunability of $\mathrm{CsPbBr}_{3} @ \mathrm{KBr}$ NCCs by variation of $\mathrm{KBr}_{3} \mathrm{CsPbBr}_{3} \mathrm{molar}$ ratio; the average size of LHP NCs progressively decreases with increasing carrier:LHP ratio. (b) PL QY dependence on carrier:LHP ratio for the $\mathrm{CsPbBr}_{3} @ \mathrm{KBr}$ system. The highlighted areas correspond to different regimes: orange, coexistence of LHP NCs and bulkier LHP microcrystals that reabsorb emitted light; green, the optimal range, where LHPs are mainly present in the form of large NCs (weak confinement regime or without quantum confinement); and blue, LHP NCs are small and exhibit medium or strong quantum confinement. (c) Time-resolved PL spectra of $\mathrm{CsPbBr}_{3} @ \mathrm{KBr}$ NCCs with various $\mathrm{KBr}: \mathrm{CsPbBr}_{3}$ ratios; the pumping intensity $10 \mathrm{~nJ} / \mathrm{cm}^{2}$. (d) A photograph depicting various NCCs dispersed in a variety of containers such as plastic balls, glass dishes, and capillaries to demonstrate the visual tunability of their emission color through the size and composition of LHP NCs.

the carrier. XRD measured at a synchrotron light source (SLS $\mathrm{XRD)}$ reveals the broad modulation of the background that coincides with $\mathrm{CsPbBr}_{3}$ (Figure S5, see SI for details). This modulation indicates that $\mathrm{CsPBr}_{3}$ is present in the form of NCs with sizes roughly estimated to be below $10 \mathrm{~nm}$, similar to the NCs seen in the STEM images.

The variation of the carrier:LHP ratio is the main tool for controlling this synthetic process. An increasing ratio changes the area available for the heterogeneous nucleation of LHP NCs and therefore the number of nuclei. This allowed us to tune the average size of LHP NCs, which can be roughly determined from the PL spectra for medium to small LHP NCs by using a sizing curve reported earlier for colloidal $\mathrm{CsPbBr}_{3}$ NCs (Figures 2a and S6). ${ }^{43}$ At high LHP loading $\left(\mathrm{KBr}: \mathrm{CsPbBr}_{3}\right.$ ratios <20:1), the PL maximum is located at around $520 \mathrm{~nm}$, indicating the presence of large LHP NCs $(>20 \mathrm{~nm})$. However, the number of available sites for heterogeneous nucleation is limited, and an additional impurity of orange, non-luminescent microcrystals of $\mathrm{CsPbBr}_{3}$ can also be observed (Figure S7). At slightly lower LHP loading ( $\mathrm{KBr}: \mathrm{Cs} \mathrm{PbBr}_{3}$ molar ratio from $20: 1$ to $50: 1$ ), this impurity disappears, yet the PL peak position remains nearly unchanged in the range of 515-520 nm (corresponds to $\sim 14-20 \mathrm{~nm}$ large NCs). Even lower LHP loading ( $\mathrm{KBr}: \mathrm{CsPbBr}_{3}$ ratio above 50:1) leads to an insufficient amount of precursors. In this case, the diffusion-limited growth of $\mathrm{CsPbBr}$ NCs results in a decrease of the average size and a blueshift of the PL maximum to $495 \mathrm{~nm}$, which corresponds roughly to $7 \mathrm{~nm}$ large NCs (Figures 2a and S6). For various carriers, the PL starts to blueshift at slightly different LHP:carrier ratios (Figure S6). At very low LHP loading ( $\mathrm{KBr}: \mathrm{CsPBBr}_{3}$ ratios from 100:1 to 1000:1), the PL further shifts to $480 \mathrm{~nm}(\sim 5 \mathrm{~nm} \mathrm{NCs})$, and a fraction of pure $\mathrm{KBr}$ microcrystals with perfect tetrahedral habits and without PL can be observed (Figure S8).

Despite the lack of ligands or other additives that traditionally control the nucleation and growth of NCs, these
NCs still exhibit relatively narrow PL in the weak confinement regime (PL peak position at $>515 \mathrm{~nm}$ corresponding to NCs with sizes above $14 \mathrm{~nm}$ ). A typical PL full-width-at-halfmaximum (fwhm) for green-emitting powders is about 20-22 $\mathrm{nm}$ for $\mathrm{CsPbBr}_{3}$ and $25-27 \mathrm{~nm}$ for $\mathrm{FAPbBr}_{3}$. This is comparable to the fwhm of colloidal ${ }^{1,12}$ and templated ${ }^{8}$ LHP NCs and much narrower than that of state-of-the-art green Cdfree NC emitters. ${ }^{93,94}$ We note that such narrow emission is one of the key factors required for color pure Rec.2020 standards for next-generation displays. ${ }^{95}$

As mentioned above, the dilution of $\mathrm{CsPbBr}{ }_{3} \mathrm{NCs}$ down to a carrier:LHP molar ratio of 50:1 does not change the wavelength of the PL maximum. However, this dilution significantly improves the PL QY of unshelled NCCs (Figure $2 \mathrm{~b})$. In the case of a high LHP loading $(\mathrm{KBr}: \mathrm{CsPbBr}$ ratio below 10:1), the formation of $\mathrm{CsPbr}_{3} \mathrm{NCs}$ on $\mathrm{KBr}$ carriers competes with the growth of bulk $\mathrm{CsPBr}_{3}$ in the form of microcrystals (Figure S7). These $\mathrm{CsPbBr}_{3}$ microcrystals are not luminescent, and they absorb light from the excitation source and decrease the overall PL QY of the material. Increasing the $\mathrm{KBr}: \mathrm{CsPbBr}_{3}$ molar ratio decreases the average size of $\mathrm{CsPbBr}_{3}$ NCs and minimizes the amount of bulk $\mathrm{CsPbBr}{ }_{3}$ microcrystals. At a $\mathrm{KBr}: \mathrm{CsPbBr}_{3}$ molar ratio of 30:1, such microcrystals were no longer observed in optical microscopy, and the measured PL QY was about $55 \pm 15 \%$. A further increase in the carrier:LHP ratio leads to the dilution of $\mathrm{CsPbBr}_{3} \mathrm{NCs}$ and further increased the PL QY of the unshelled NCCs to $68 \pm 24 \%$. The optimal concentration, however, depends on the LHP composition and its carrier. The optimal carriers for $\mathrm{CsPbBr}_{3}$ and $\mathrm{FAPbBr}_{3}$ were $\mathrm{KBr}$ and $\mathrm{NaBr}$, respectively. Other carriers produce LHP@carrier composites with $\sim 10$ times lower brightness. The brightest $\mathrm{CsPbr}_{3} @ \mathrm{KBr}$ NCCs were obtained at a $\mathrm{KBr}: \mathrm{CsPbBr}_{3}$ ratio of 40:1; whereas for the $\mathrm{FAPbBr}_{3} @ \mathrm{NaBr}$ system, the optimal ratio was 20:1. The best samples exhibit PL QY as high as $89 \%$ for $\mathrm{CsPbB}_{3}$ and $83 \%$ for $\mathrm{FAPbBr}_{3}$. 
Within the range where the PL QY varies slightly (40:1 to 100:1 carrier:LHP ratio), the PL shifts from 520 to $495 \mathrm{~nm}$ and the PL decay becomes $3.5 \times$ faster. This trend is in agreement with the PL lifetime dependence on NC size that has been reported earlier for colloidal $\mathrm{CsPbBr}_{3} \mathrm{NCs}$ : smaller $\mathrm{NCs}$ have faster radiative relaxation rates at room temperature than larger ones. ${ }^{96}$ At the same time, we found the PL decay rate to be nearly independent of the carrier if NCs of a similar size are compared (Figure S9).

Although all of the studied alkali-halides behave similarly as carriers, CsX cannot be easily compared to the others due to the presence of intermediate compounds in the $\mathrm{CsX}-\mathrm{PbX}_{2}$ system. Instead, these intermediate compounds can themselves be used as carriers. This extends the concept of concomitant iso-anionic crystallization of LHP NCs and carriers to a fully iso-atomic system, where $\mathrm{CsPbX}_{3} \mathrm{NCs}$ are grown on $\mathrm{Cs}_{4} \mathrm{PbX}_{6}$ based carriers. The pseudobinary $\mathrm{CsBr}-\mathrm{PbBr}_{2}$ system contains three compounds: $\mathrm{CsPbBr}_{3}, \mathrm{CsPb}_{2} \mathrm{Br}_{5}$, and $\mathrm{Cs}_{4} \mathrm{PbBr}_{6} \cdot{ }^{97}$ The last two melt incongruently, indicating that the formation of $\mathrm{Cs} \mathrm{PbBr}_{3}$ and binary parent compounds is more energetically favorable (Figure S10). The formation of $\mathrm{CsPbBr}_{3} \mathrm{NCs}$ on the surface of $\mathrm{Cs}_{4} \mathrm{PbBr}_{6}$ can, however, be favored by the fact that these two phases have very similar atomic positions and symmetry along several planes. ${ }^{76}$ The optical properties of the resulting $\mathrm{CsPbX}_{3} @ \mathrm{Cs}_{4} \mathrm{PbX}_{6}$ powders are similar to those synthesized on alkali halide carriers. By adjusting the anion composition in $\mathrm{CsPbX}_{3} @ \mathrm{Cs}_{4} \mathrm{PbX}_{6} \mathrm{NCCs}$, the emission wavelength can be tuned between $400 \mathrm{~nm}$ (corresponding to $\mathrm{CsPCl}_{3}$ ) and $700 \mathrm{~nm}$ (corresponding to $\mathrm{CsPbI}_{3}$ ), as shown in Figure S11. Only partial anion exchange was possible as compositions with $\sim 15-50 \%$ of heavier anion show two PL bands indicating phase separation, analogous to previous reports for the bulk $\mathrm{CsPbBr}{ }_{3}-\mathrm{CsPb}_{3}$ system. For alkali-based matrices, the range where solid solutions were unstable was generally broader, and only $\sim 20 \%$ of the heavier halide could be exchanged with a lighter one for both the $\mathrm{Br} / \mathrm{Cl}$ and $\mathrm{I} / \mathrm{Br}$ systems. Despite the appealing optical properties of $\mathrm{CsPbX}_{3} @$ $\mathrm{Cs}_{4} \mathrm{PbX}_{6}$ NCCs, we did not consider them for further investigation because the $\mathrm{Cs}_{4} \mathrm{PbX}_{6}$ carrier significantly increases the overall $\mathrm{Pb}$ content of the composite material. Instead, only NCCs grown on $\mathrm{Pb}$-free carriers have been shelled with salt matrixes.

While anchoring LHP NCs to the carrier surface minimizes the probability of NC merging, it does not protect NCs from their environment. In order to fully encapsulate NCs into salt matrices, we grew an additional alkali halide shell on the NCC microcrystals. To do so, we developed a polar-solvent-free shelling technique based on the reaction of amphiphilic $\mathrm{Na}^{+}$ and $\mathrm{Br}^{-}$salts, namely sodium docusate (AOT) and didodecylammonium bromide (DDAB) in toluene. Both salts are highly soluble and slowly react in toluene, leading to the precipitation of $\mathrm{NaBr}$ over the course of $12 \mathrm{~h}$. The introduction of $\mathrm{Na}, \mathrm{K}$, or Rb-based NCCs into this system results in the heterogeneous nucleation and growth of $\mathrm{NaBr}$ around the NCC, resulting in the final NCC/ $\mathrm{NaBr}$ (Scheme 1). These shelled microcrystals can be obtained in the form of fairly stable dispersions (Figure 3a) or powders if all surfactants are washed away (Figure $3 b$ ). The shelled NCC/ $\mathrm{NaBr}$ exhibit a random non-uniform spatial distribution of emission centers inside the microcrystal (Figure 3c). This is in stark contrast to the unshelled NCCs, where LHP NCs are localized primarily on the surface (Figure $1 \mathrm{~b}, \mathrm{e}, \mathrm{f}$ ). This fact, together with the clearly polycrystalline nature of the shelled

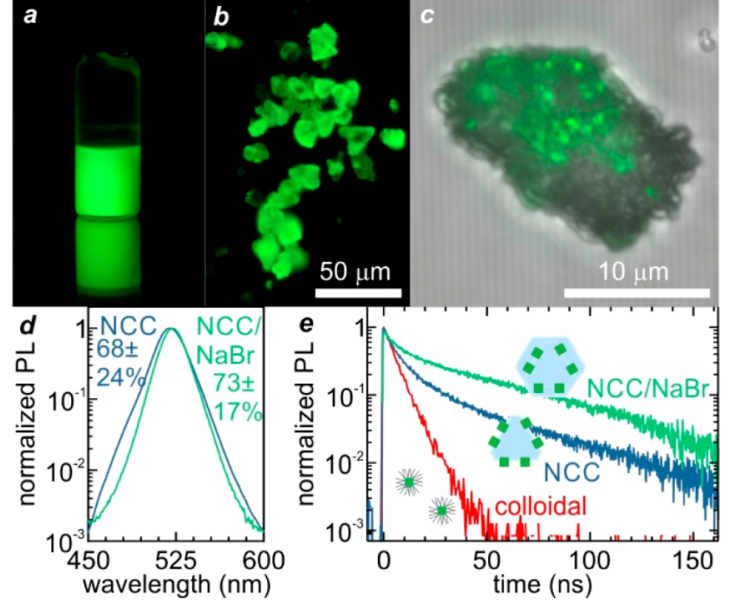

Figure 3. Shelled $\mathrm{CsPbBr}_{3} @ \mathrm{KBr} / \mathrm{NaBr}$ microcrystals: (a) suspension in toluene under UV light and (b) optical and (c) confocal microscopy. (d) Comparison of PL spectrum of initial CsPbBr $\mathrm{Cr}_{3} @$ $\mathrm{KBr} \mathrm{NCC}$ (blue curve) and shelled $\mathrm{NCC} / \mathrm{NaBr}$ (green curve). (e) Time-resolved PL of $\mathrm{CsPbBr}_{3} @ \mathrm{KBr}$ NCC and shelled $\mathrm{CsPbBr}_{3} @$ $\mathrm{KBr} / \mathrm{NaBr}$ in comparison with colloidal $\mathrm{CsPbBr}_{3} \mathrm{NCs}$.

microcrystals, indicates that the $\mathrm{NaBr}$ grows heterogeneously on the surface of NCCs but not separately.

The dissolution and merging of LHP NCs could have potentially been the main issues encountered upon treating NCs with polar DDAB and AOT species, and this was indeed the case when colloidal $\mathrm{CsPbr}_{3}$ NCs were subjected to an analogous shelling procedure. In contrast, the anchored NCCs remain stable and similarly bright after shelling. The PL peak position remains nearly unchanged (Figure $3 \mathrm{~d}$ ), and the PL fwhm slightly narrows. This may be primarily due to the growth of any small LHP NCs on the surface, which pushes them toward weaker quantum confinement (Figure 3d). The overall stability of the PL peak position and profile indicates that anchored NCCs do not dissolve during shelling procedure. On average, the PL QY remains unchanged or it even slightly increases (Figure 3d). Furthermore, the average measured lifetime increases from about $25 \mathrm{~ns}$ for optimal $\mathrm{CsPbBr}_{3}$ NCCs to $45 \mathrm{~ns}$ for shelled $\mathrm{CsPbBr}_{3} \mathrm{NCC} / \mathrm{NaBr}$ (Figure $3 \mathrm{e}$ ).

In order to check if anchored LHP NCs can merge during the shelling procedure, we investigated the $\mathrm{PL}$ temperature dependence of the final, shelled $\mathrm{NCC} / \mathrm{NaBr}$ samples (Figure 4). At room temperature, the PL of bulk $\mathrm{CsPbBr}$ is very weak and cannot be detected in the presence of bright NCs. However, the PL of bulk CsPbBr 3 impurities, if present, can be well resolved at $4-15 \mathrm{~K}$ when it becomes much brighter and narrower with a peak position at $539 \mathrm{~nm}$ (Figures S12). We have found that the PL of bulk impurities can be readily differentiated from the PL of even very large NCs which emit at $525 \mathrm{~nm}$ at room temperature and are expected to be above $30 \mathrm{~nm}$ in size (Figures S12 and S13). ${ }^{43}$ Our optimized $\mathrm{CsPbBr}_{3} @ \mathrm{KBr} / \mathrm{NaBr}$ powder exhibits PL at $520 \mathrm{~nm}$ at room temperature and therefore should contain smaller LHP NCs. This allowed us to resolve any potential impurity of bulk $\mathrm{CsPbBr}$ if it would have been present in the sample. Figure $4 \mathrm{a}$ indicates that the PL spectrum collected at $15 \mathrm{~K}$ does not show a sharp line at a wavelength of $539 \mathrm{~nm}(2.300 \mathrm{eV})$. This indicates that our anchored LHP NCs do not merge (at least not to the point that they grow above $30 \mathrm{~nm}$ in size) during the shelling process. 

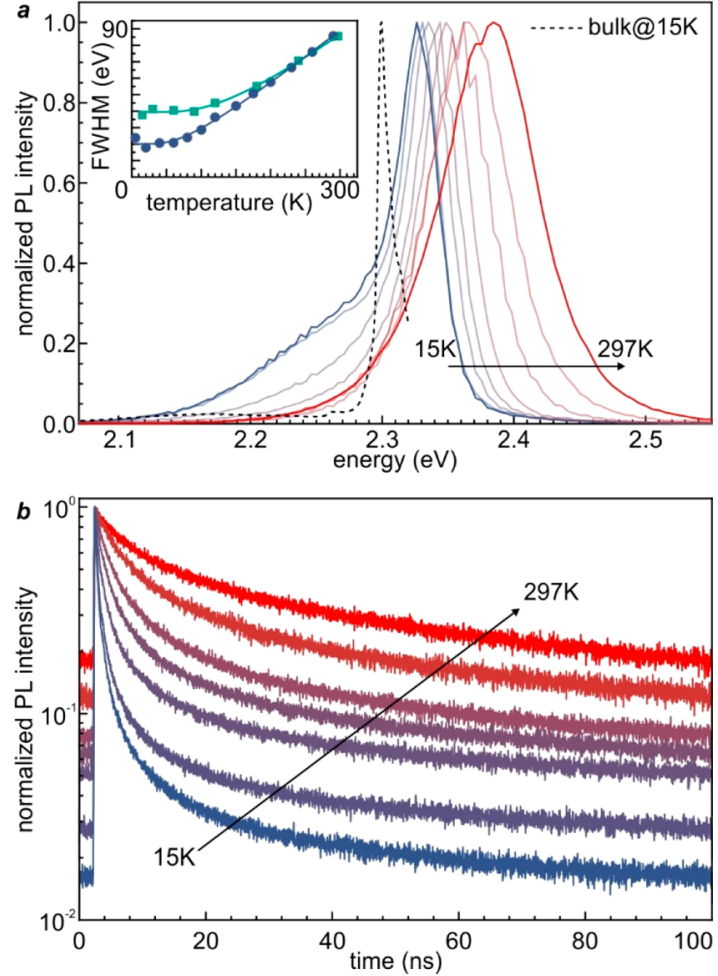

Figure 4. (a) PL dependence on temperature for shelled $\mathrm{CsPbBr}_{3} @ \mathrm{KBr} / \mathrm{NaBr}$. The dashed line shows PL spectrum of bulk $\mathrm{CsPbBr}_{3}$ at $15 \mathrm{~K}$. Inset shows $\mathrm{PL}$ fwhm dependence on temperature for smaller $\mathrm{CsPbBr}_{3} @ \mathrm{KBr} / \mathrm{NaBr}$ ( $\mathrm{PL}$ maximum at $520 \mathrm{~nm}$ at room temperature, green symbols) and very large $\mathrm{CsPbBr}_{3} @ \mathrm{Cs}_{4} \mathrm{PbBr}_{6}$ (PL maximum at $525 \mathrm{~nm}$ at room temperature, blue symbols). (b) Time-resolved PL spectra of shelled $\mathrm{CsPbBr}_{3} @ \mathrm{KBr} / \mathrm{NaBr}$ microcrystals at various temperatures.

The overall PL dependence on temperature for the shelled LHPs NCs is very similar to that of colloidal LHP NCs. ${ }^{7}$ The $\mathrm{PL}$ redshifts to $2.331 \mathrm{eV}$ (532 $\mathrm{nm})$ upon cooling to $15 \mathrm{~K}$ and narrows down to $20-40 \mathrm{meV}$. This fwhm indicates that the size distribution of $\mathrm{CsPbBr}_{3} \mathrm{NCs}$ is roughly two times broader than the best colloidal counterparts. ${ }^{96}$ The fast component of the radiative decay rate accelerates from about 10 to $0.6 \mathrm{~ns}$ at $15 \mathrm{~K}$. Those values are typical for large $(15-20 \mathrm{~nm})$ colloidal LHP NCs. ${ }^{10,96}$

In addition to preserving or even enhancing the optical properties of LHP NCs, the fully shelled LHP NCCs/ $\mathrm{NaBr}$ also demonstrate good stability against many polar solvents. They withstand sonication in pure acetone, acetonitrile, ethanol, $N$-methylpyrrolidone, $\gamma$-butyrolactone, tetramethylurea, and triethylamine (Figure 5). In contrast, colloidal LHP NCs either completely dissolve or merge even in the presence of minor quantities of polar molecules ( $9 \mathrm{vol} \%$ in toluene) and consequently suffer a significant decrease in their PL QY. The shelled NCCs $/ \mathrm{NaBr}$ instead dissolve only slightly in some solvents and retain their high PL QY. We attribute the enhanced stability to the fact that the aforementioned solvents, while able to dissolve or degrade $\mathrm{LHPs}^{98}$ are rather poor solvents for alkali halides. As a result, only fully shelled LHP NCCs can survive treatments with such solvents, whereas poorly shelled or unshelled ones dissolve. This aspect can be used to wash out poorly shelled NCCs from the ones which are properly shelled.

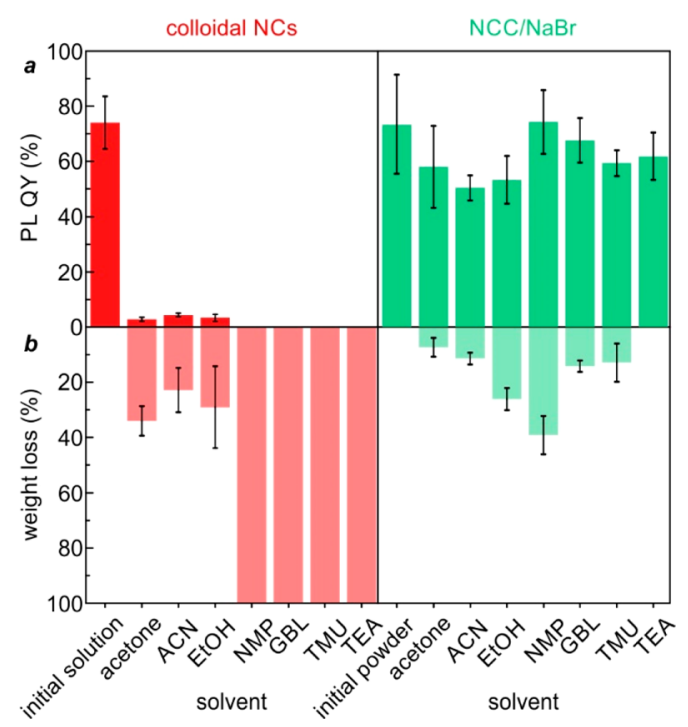

Figure 5. Stability of colloidal CsPbBr ${ }_{3} \mathrm{NCs}$ and shelled CsPbBr $\mathrm{C}_{3} @$ $\mathrm{KBr} / \mathrm{NaBr}$ microcrystals against select polar solvents. The decreased PL QY (a) is ascribed to the degree of merging of LHP NCs in each case. The weight loss for materials treated with the corresponding solvent is shown in (b). In all cases, the total amount of LHP was kept constant at $0.1 \mathrm{mg}$. For colloidal NCs, only a small amount of the polar solvent $(9 \mathrm{vol} \%)$ was added to the toluene solution. For $\mathrm{NCC} / \mathrm{NaBr}$, the powders were sonicated for $5 \mathrm{~min}$ in $200 \mu \mathrm{L}$ of the pure, polar solvent. A $100 \%$ weight loss in the case of colloidal NCs indicates the complete dissolution of the LHP NCs treated with NMP, GBL, TMU, and TEA.

Given that shelling protects LHP NCCs from attack by many polar molecules and stabilizes them against merging, one can now use these powders in many post-synthetic processes that would otherwise diminish the optical properties of colloidal LHP NCs. For example, one can perform polymerization with numerous monomers or embed these microcrystals into polymers without significant loss of PL QY (Figure $6 a-c)$. As there are many polymers with very low water and oxygen permeability and many of them can be used as an encapsulant for such materials, we chose to investigate those which could further enhance the stability of these shelled LHP NCCs. Therefore, polystyrene, cyclic olefin copolymer TOPAS, and various UV-curable monomers have been tested including methyl methacrylate, lauryl methacrylate, silicon urethane acrylate (Miramer SIU2400), bisphenol A glycerolate diacrylate, NOA 63 with or without additives of ethylene glycol dimethacrylate, 2-hydroxyethyl methacrylate, vinyl acetate, and isobornyl acrylate. In all of these cases, the PL QY of NCC/ $\mathrm{NaBr}$ did not drop after embedding them into a polymer encapsulant. This is again in stark contrast to colloidal $\mathrm{CsPbBr}_{3}$ NCs which are known to often lose their PL QY after being embedded into polymers. ${ }^{12}$

At elevated temperatures, all samples show decreased PL QY, which is an intrinsic property of LHPs. ${ }^{99}$ Shelled $\mathrm{FAPbBr}_{3}$ NCs exhibit the best thermal stability (Figure 6d,e). At 40-50 ${ }^{\circ} \mathrm{C}$, which is a typical operating temperature for backlit displays, their PL intensity only decreases by about 5\%. At 120 ${ }^{\circ} \mathrm{C}$, the PL intensity decreases by $40 \%$. However, we found that briefly heating $\left(\sim 20 \mathrm{~min}\right.$ at temperatures above $\left.100{ }^{\circ} \mathrm{C}\right)$ does not permanently degrade the samples; the PL intensity fully recovers when samples are cooled down to room temperature (Figure 6d). Similarly, the PL peak position blue shifts by about $8 \mathrm{~nm}$ upon heating to $120^{\circ} \mathrm{C}$ but returns to its original 

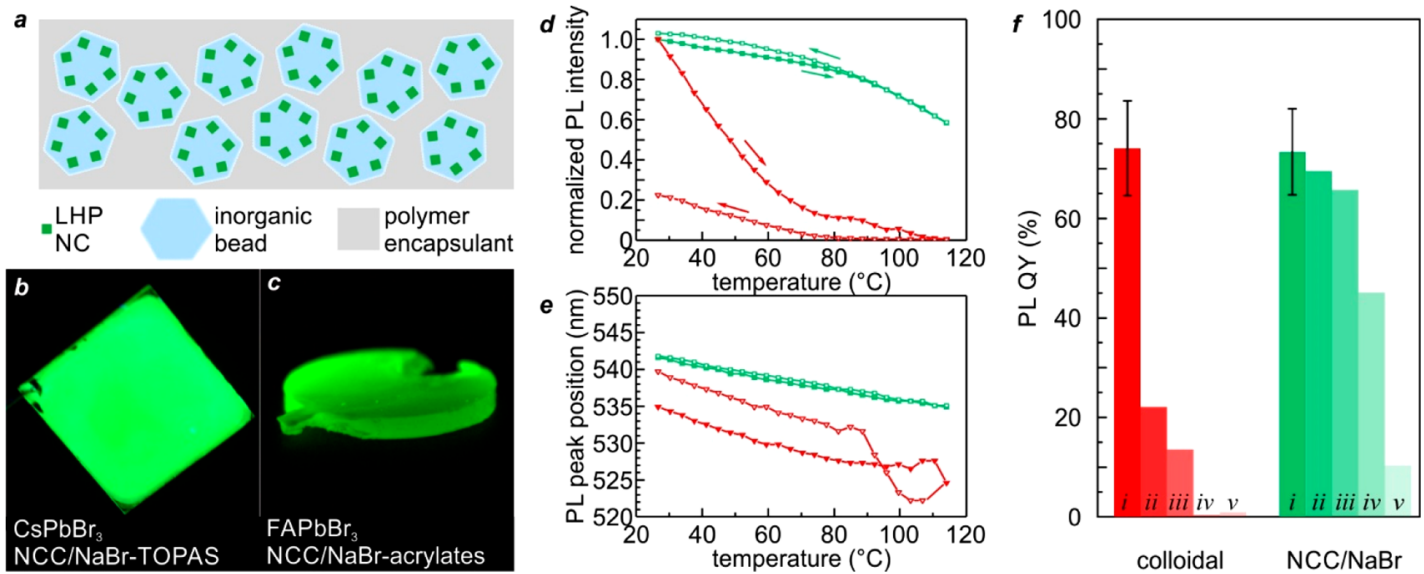

Figure 6. Stability of NaBr-shelled LHP NCCs. (a) Scheme of the samples used for reliability tests: NaBr-shelled LHP NCCs are embedded into a polymer encapsulant. The polymer encapsulant is prepared either by drying the polymer solution (b) or by UV-curing a mixture of monomers (c). PL intensity (d) and peak position (e) change during heating to $120{ }^{\circ} \mathrm{C}$ (closed symbols) followed by cooling to room temperature (opened symbols) for shelled $\mathrm{FAPbBr}_{3} \mathrm{NCC} / \mathrm{NaBr}$ (green) and colloidal $\mathrm{FAPbBr}_{3} \mathrm{NCs}$ (red curve). (f) PL QY drops after 190 $h$ in three different tests of shelled $\mathrm{CsPbBr}_{3} \mathrm{NCC} / \mathrm{NaBr}$ in comparison with colloidal $\mathrm{CsPbBr}_{3} \mathrm{NCs}$ : (i) initial sample, (ii) $\mathrm{NCs}$ embedded in acrylate film, (iii) reliability test at $95 \% \mathrm{RH}$ and $50{ }^{\circ} \mathrm{C}$, (iv) thermal stability test at $80{ }^{\circ} \mathrm{C}$, and (v) thermal stability test at $120{ }^{\circ} \mathrm{C}$.

position after cooling (Figure 6e). Additional heating/cooling cycles can be repeated with complete reversibility. In contrast, colloidal NCs embedded into polymer films do not exhibit this thermal stability and instead quickly merge (Figures 6d,e).

In order to verify the long-term stability of $\mathrm{NCC} / \mathrm{NaBr}$ microcrystals, we embedded them into polymer films and performed three reliability tests: (i) at $80{ }^{\circ} \mathrm{C}$, (ii) at $120{ }^{\circ} \mathrm{C}$, and (iii) at high humidity and elevated temperature $(95 \% \mathrm{RH}$, $50{ }^{\circ} \mathrm{C}$ ). These tests were performed in notably harsher conditions than required for real display applications. This allowed us to probe the material degradation in an accelerated regime. After $190 \mathrm{~h}$ under such harsh conditions, the shelled $\mathrm{CsPbBr}{ }_{3} \mathrm{NCC} / \mathrm{NaBr}$ samples showed notably higher stability compared to colloidal NCs (Figure 6f): about $65 \%$ of the initial PL QY remained after $190 \mathrm{~h}$ at $80{ }^{\circ} \mathrm{C}$, and the PL QY drop during the humidity test was negligible (although the overall brightness dropped a bit, probably due to partial diffusion of water inside the polymer.) To the best of our knowledge, this is the best stability reported so far for LHP NCs, and it is enabled by the combination of a robust inorganic matrix with a polymer encapsulant.

\section{CONCLUSIONS}

In summary, we propose a two-step approach to the synthesis of LHP NCs embedded into robust Pb-free microcrystalline, inorganic salt matrices. The key point of this approach is the initial concomitant crystallization of LHP NCs and salt carriers. By anchoring LHP NCs to the carrier, they are stabilized against merging and can be coated with an optically transparent inorganic shell. For this second step, we developed a method that involves the direct reaction of amphiphilic $\mathrm{Na}$ and $\mathrm{Br}$ precursors in polar-solvent-free media, resulting in the precipitation of $\mathrm{NaBr}$ on the surface of salt carriers and LHP NCs. This results in NaBr-shelled-NCCs with optical properties on par with the best colloidal LHP NCs, including high PL QY of $\sim 90 \%$, pure PL colors with line widths as narrow as 20 $\mathrm{nm}$ for green LHPs, and PL tunability through both NC size and anion composition. At the same time, the stability of these powders toward polar moieties, such as ethanol, acetonitrile, $\gamma$ butyrolactone, or acrylates, is significantly improved due to the complete isolation of LHP NCs from each other and from the environment. This permits the use of shelled LHP NCs in processes which otherwise degrade the optical properties of colloidal LHP NCs. Finally, the introduction of non-absorbing inorganic shells and matrices increases their overall scattering to facilitate improved out-coupling efficiencies for downconverting films in LCDs.

\section{EXPERIMENTAL SECTION}

Synthesis of LHP NCs on Alkali Halide Carriers. The relevant solutions from Table S1 were mixed in a desired ratio, homogenized by vortex mixing, drop-casted onto a glass substrate, and heated to 70 ${ }^{\circ} \mathrm{C}$ for $5 \mathrm{~h}$ (for $\mathrm{Cl}$ - and $\mathrm{Br}$-based compositions) or to $200{ }^{\circ} \mathrm{C}$ for 10 min (for I-based compositions). For $\mathrm{FAPbX}_{3}$ NCCs $(\mathrm{X}=\mathrm{Br}, \mathrm{I})$, an excess of FAX in respect to $\mathrm{PbX}_{2}$ was used.

Shelling of NCs-on-Carrier (NCC) with $\mathrm{NaBr}$. NCC powders were thoroughly ground or mildly ball-milled. The obtained fine powder $(10 \mathrm{mg})$ was dispersed in $2 \mathrm{~mL}$ of a DDAB solution in toluene $(0.5 \mathrm{M})$ and sonicated for $5 \mathrm{~min}$. The resulting dispersion was stirred, while $0.5 \mathrm{~mL}$ of an AOT solution in toluene $(2 \mathrm{M})$ was added dropwise over the course of an hour. The reaction mixture was kept stirring for the next $12 \mathrm{~h}$. The final dispersion was washed with toluene and ethyl acetate, dried in vacuum, and stored inside a glovebox.

Encapsulation of $\mathrm{NCC}$ and $\mathrm{NCC} / \mathrm{NaBr}$ into PS or TOPAS Polymers. The desired amount of $\mathrm{NCC} / \mathrm{NaBr}$ powder was dispersed in a polymer solution in toluene $(10 \mathrm{wt} \%)$ and cast onto a glass substrate and dried at $50{ }^{\circ} \mathrm{C}$ for $1 \mathrm{~h}$.

Example of the Encapsulation of $\mathrm{NCC} / \mathrm{NaBr}$ into UV-Cured Polymers. The desired amount of NCC/NaBr powder was added to a distilled LMA:EGDMA mixture (4:1) with $0.1 \%$ of Irgacure 819 . The obtained mixture was cast onto a glass substrate and cured for 15 min under $20 \mathrm{~mW} / \mathrm{cm}^{2}$ flux $(365 \mathrm{~nm})$.

\section{ASSOCIATED CONTENT}

\section{S Supporting Information}

The Supporting Information is available free of charge on the ACS Publications website at DOI: 10.1021/acsnano.9b05481.

Details of the materials synthesis, encapsulation into polymer matrices, characterization techniques and stability tests, additional supplementary figures, optical microphotographs, confocal and electron microscopy 
images, EDXS and XRD data, TR PL, and temperaturedependent PL spectra (PDF)

\section{AUTHOR INFORMATION}

\section{Corresponding Author}

*E-mail: mvkovalenko@ethz.ch. ORCID $\odot$

Dmitry N. Dirin: 0000-0002-5187-4555

Sergii Yakunin: 0000-0002-6409-0565

Frank Krumeich: 0000-0001-5625-1536

Maksym V. Kovalenko: 0000-0002-6396-8938

Notes

The authors declare no competing financial interest.

\section{ACKNOWLEDGMENTS}

We thank the European Union for financial support via ERC Starting Grant 2012 (Project NANOSOLID, GA no. 306733) and Swiss Federal Commission for Technology and Innovation for financial support (CTI-no. 18614.1 PFNM-NM). We also acknowledge Marie Curie Fellowship (IIF-GA-2012-330524) and Marie Curie ITN network PHONSI (H2020-MSCA-ITN642656). Authors thank IBM-Zurich Research, in particular Dr. T. Stoferle and Dr. R. F. Mahrt, for support with lowtemperature PL experiments. We also acknowledge Scope M (ETH Zurich) and Dr. Simona Rodighiero for the help with confocal microscopy.

\section{REFERENCES}

(1) Protesescu, L.; Yakunin, S.; Bodnarchuk, M. I.; Krieg, F.; Caputo, R.; Hendon, C. H.; Yang, R. X.; Walsh, A.; Kovalenko, M. V. Nanocrystals of Cesium Lead Halide Perovskites $\left(\mathrm{CsPbX}_{3}, \mathrm{X}=\mathrm{Cl}, \mathrm{Br}\right.$, and I): Novel Optoelectronic Materials Showing Bright Emission with Wide Color Gamut. Nano Lett. 2015, 15, 3692-3696.

(2) Huang, H.; Bodnarchuk, M. I.; Kershaw, S. V.; Kovalenko, M. V.; Rogach, A. L. Lead Halide Perovskite Nanocrystals in the Research Spotlight: Stability and Defect Tolerance. ACS Energy Lett. 2017, 2, 2071-2083.

(3) Akkerman, Q. A.; Rainò, G.; Kovalenko, M. V.; Manna, L. Genesis, Challenges and Opportunities for Colloidal Lead Halide Perovskite Nanocrystals. Nat. Mater. 2018, 17, 394-405.

(4) Yang, D.; Li, X.; Zeng, H. Surface Chemistry of All Inorganic Halide Perovskite Nanocrystals: Passivation Mechanism and Stability. Adv. Mater. Interfaces 2018, 5, 1701662.

(5) Wang, H.-C.; Bao, Z.; Tsai, H.-Y.; Tang, A.-C.; Liu, R.-S. Perovskite Quantum Dots and Their Application in Light-Emitting Diodes. Small 2018, 14, 1702433.

(6) Sun, S.; Yuan, D.; Xu, Y.; Wang, A.; Deng, Z. Ligand-Mediated Synthesis of Shape-Controlled Cesium Lead Halide Perovskite Nanocrystals Via Reprecipitation Process at Room Temperature. ACS Nano 2016, 10, 3648-3657.

(7) Rainò, G.; Nedelcu, G.; Protesescu, L.; Bodnarchuk, M. I.; Kovalenko, M. V.; Mahrt, R. F.; Stöferle, T. Single Cesium Lead Halide Perovskite Nanocrystals at Low Temperature: Fast SinglePhoton Emission, Reduced Blinking, and Exciton Fine Structure. ACS Nano 2016, 10, 2485-2490.

(8) Dirin, D. N.; Protesescu, L.; Trummer, D.; Kochetygov, I. V.; Yakunin, S.; Krumeich, F.; Stadie, N. P.; Kovalenko, M. V. Harnessing Defect-Tolerance at the Nanoscale: Highly Luminescent Lead Halide Perovskite Nanocrystals in Mesoporous Silica Matrixes. Nano Lett. 2016, 16, 5866-5874.

(9) Rainò, G.; Becker, M. A.; Bodnarchuk, M. I.; Mahrt, R. F.; Kovalenko, M. V.; Stöferle, T. Superfluorescence from Lead Halide Perovskite Quantum Dot Superlattices. Nature 2018, 563, 671-675.

(10) Becker, M. A.; Vaxenburg, R.; Nedelcu, G.; Sercel, P. C.; Shabaev, A.; Mehl, M. J.; Michopoulos, J. G.; Lambrakos, S. G.;
Bernstein, N.; Lyons, J. L.; Stöferle, T.; Mahrt, R. F.; Kovalenko, M. V.; Norris, D. J.; Rainò, G.; Efros, A. L. Bright Triplet Excitons in Caesium Lead Halide Perovskites. Nature 2018, 553, 189.

(11) Tamarat, P.; Bodnarchuk, M. I.; Trebbia, J.-B.; Erni, R.; Kovalenko, M. V.; Even, J.; Lounis, B. The Ground Exciton State of Formamidinium Lead Bromide Perovskite Nanocrystals Is a Singlet Dark State. Nat. Mater. 2019, 18, 717-724.

(12) Protesescu, L.; Yakunin, S.; Bodnarchuk, M. I.; Bertolotti, F.; Masciocchi, N.; Guagliardi, A.; Kovalenko, M. V. Monodisperse Formamidinium Lead Bromide Nanocrystals with Bright and Stable Green Photoluminescence. J. Am. Chem. Soc. 2016, 138, 1420214205.

(13) Protesescu, L.; Yakunin, S.; Kumar, S.; Bär, J.; Bertolotti, F.; Masciocchi, N.; Guagliardi, A.; Grotevent, M.; Shorubalko, I.; Bodnarchuk, M. I.; Shih, C.-J.; Kovalenko, M. V. Dismantling the "Red Wall" of Colloidal Perovskites: Highly Luminescent Formamidinium and Formamidinium-Cesium Lead Iodide Nanocrystals. ACS Nano 2017, 11, 3119-3134.

(14) Pfingsten, O.; Klein, J.; Protesescu, L.; Bodnarchuk, M. I.; Kovalenko, M. V.; Bacher, G. Phonon Interaction and Phase Transition in Single Formamidinium Lead Bromide Quantum Dots. Nano Lett. 2018, 18, 4440-4446.

(15) Levchuk, I.; Osvet, A.; Tang, X.; Brandl, M.; Perea, J. D.; Hoegl, F.; Matt, G. J.; Hock, R.; Batentschuk, M.; Brabec, C. J. Brightly Luminescent and Color-Tunable Formamidinium Lead Halide Perovskite $\mathrm{FAPbX}_{3}(\mathrm{X}=\mathrm{Cl}, \mathrm{Br}, \mathrm{I})$ Colloidal Nanocrystals. Nano Lett. 2017, 17, 2765-2770.

(16) Akkerman, Q. A.; D’Innocenzo, V.; Accornero, S.; Scarpellini, A.; Petrozza, A.; Prato, M.; Manna, L. Tuning the Optical Properties of Cesium Lead Halide Perovskite Nanocrystals by Anion Exchange Reactions. J. Am. Chem. Soc. 2015, 137, 10276-10281.

(17) Nedelcu, G.; Protesescu, L.; Yakunin, S.; Bodnarchuk, M. I.; Grotevent, M. J.; Kovalenko, M. V. Fast Anion-Exchange in Highly Luminescent Nanocrystals of Cesium Lead Halide Perovskites ( $\left.\mathrm{CsPbX}_{3}, \mathrm{X}=\mathrm{Cl}, \mathrm{Br}, \mathrm{I}\right)$. Nano Lett. 2015, 15, 5635-5640.

(18) Zhang, D.; Yang, Y.; Bekenstein, Y.; Yu, Y.; Gibson, N. A.; Wong, A. B.; Eaton, S. W.; Kornienko, N.; Kong, Q.; Lai, M.; Alivisatos, A. P.; Leone, S. R.; Yang, P. Synthesis of Composition Tunable and Highly Luminescent Cesium Lead Halide Nanowires through Anion-Exchange Reactions. J. Am. Chem. Soc. 2016, 138, $7236-7239$.

(19) Zhang, D.; Eaton, S. W.; Yu, Y.; Dou, L.; Yang, P. SolutionPhase Synthesis of Cesium Lead Halide Perovskite Nanowires. J. Am. Chem. Soc. 2015, 137, 9230-9233.

(20) Bekenstein, Y.; Koscher, B. A.; Eaton, S. W.; Yang, P.; Alivisatos, A. P. Highly Luminescent Colloidal Nanoplates of Perovskite Cesium Lead Halide and Their Oriented Assemblies. J. Am. Chem. Soc. 2015, 137, 16008-16011.

(21) Akkerman, Q. A.; Motti, S. G.; Srimath Kandada, A. R.; Mosconi, E.; D’Innocenzo, V.; Bertoni, G.; Marras, S.; Kamino, B. A.; Miranda, L.; De Angelis, F.; Petrozza, A.; Prato, M.; Manna, L. Solution Synthesis Approach to Colloidal Cesium Lead Halide Perovskite Nanoplatelets with Monolayer-Level Thickness Control. J. Am. Chem. Soc. 2016, 138, 1010-1016.

(22) Shamsi, J.; Dang, Z.; Bianchini, P.; Canale, C.; Di Stasio, F.; Brescia, R.; Prato, M.; Manna, L. Colloidal Synthesis of Quantum Confined Single Crystal $\mathrm{CsPbBr}_{3}$ Nanosheets with Lateral Size Control up to the Micrometer Range. J. Am. Chem. Soc. 2016, 138, $7240-7243$.

(23) Lv, L.; Xu, Y.; Fang, H.; Luo, W.; Xu, F.; Liu, L.; Wang, B.; Zhang, X.; Yang, D.; Hu, W.; Dong, A. Generalized Colloidal Synthesis of High-Quality, Two-Dimensional Cesium Lead Halide Perovskite Nanosheets and Their Applications in Photodetectors. Nanoscale 2016, 8, 13589-13596.

(24) Zhang, X.; Lv, L.; Ji, L.; Guo, G.; Liu, L.; Han, D.; Wang, B.; Tu, Y.; Hu, J.; Yang, D.; Dong, A. Self-Assembly of One-Dimensional Nanocrystal Superlattice Chains Mediated by Molecular Clusters. J. Am. Chem. Soc. 2016, 138, 3290-3293. 
(25) Koolyk, M.; Amgar, D.; Aharon, S.; Etgar, L. Kinetics of Cesium Lead Halide Perovskite Nanoparticle Growth; Focusing and De-Focusing of Size Distribution. Nanoscale 2016, 8, 6403-6409.

(26) Lignos, I.; Stavrakis, S.; Nedelcu, G.; Protesescu, L.; deMello, A. J.; Kovalenko, M. V. Synthesis of Cesium Lead Halide Perovskite Nanocrystals in a Droplet-Based Microfluidic Platform: Fast Parametric Space Mapping. Nano Lett. 2016, 16, 1869-1877.

(27) Chen, X.; Peng, L.; Huang, K.; Shi, Z.; Xie, R.; Yang, W. NonInjection Gram-Scale Synthesis of Cesium Lead Halide Perovskite Quantum Dots with Controllable Size and Composition. Nano Res. 2016, 9, 1994-2006.

(28) De Roo, J.; Ibáñez, M.; Geiregat, P.; Nedelcu, G.; Walravens, W.; Maes, J.; Martins, J. C.; Van Driessche, I.; Kovalenko, M. V.; Hens, Z. Highly Dynamic Ligand Binding and Light Absorption Coefficient of Cesium Lead Bromide Perovskite Nanocrystals. ACS Nano 2016, 10, 2071-2081.

(29) Dou, L.; Wong, A. B.; Yu, Y.; Lai, M.; Kornienko, N.; Eaton, S. W.; Fu, A.; Bischak, C. G.; Ma, J.; Ding, T.; Ginsberg, N. S.; Wang, L.W.; Alivisatos, A. P.; Yang, P. Atomically Thin Two-Dimensional Organic-Inorganic Hybrid Perovskites. Science 2015, 349, 1518-1521.

(30) Hassan, Y.; Song, Y.; Pensack, R. D.; Abdelrahman, A. I.; Kobayashi, Y.; Winnik, M. A.; Scholes, G. D. Structure-Tuned Lead Halide Perovskite Nanocrystals. Adv. Mater. 2016, 28, 566-573.

(31) Aharon, S.; Etgar, L. Two Dimensional Organometal Halide Perovskite Nanorods with Tunable Optical Properties. Nano Lett. 2016, 16, 3230-3235.

(32) Weidman, M. C.; Seitz, M.; Stranks, S. D.; Tisdale, W. A. Highly Tunable Colloidal Perovskite Nanoplatelets through Variable Cation, Metal, and Halide Composition. ACS Nano 2016, 10, 78307839.

(33) Jellicoe, T. C.; Richter, J. M.; Glass, H. F. J.; Tabachnyk, M.; Brady, R.; Dutton, S. E.; Rao, A.; Friend, R. H.; Credgington, D.; Greenham, N. C.; Böhm, M. L. Synthesis and Optical Properties of Lead-Free Cesium Tin Halide Perovskite Nanocrystals. J. Am. Chem. Soc. 2016, 138, 2941-2944.

(34) Wang, A.; Guo, Y.; Muhammad, F.; Deng, Z. Controlled Synthesis of Lead-Free Cesium Tin Halide Perovskite Cubic Nanocages with High Stability. Chem. Mater. 2017, 29, 6493-6501.

(35) Dolzhnikov, D. S.; Wang, C.; Xu, Y.; Kanatzidis, M. G.; Weiss, E. A. Ligand-Free, Quantum-Confined $\mathrm{Cs}_{2} \mathrm{SnI}_{6}$ Perovskite Nanocrystals. Chem. Mater. 2017, 29, 7901-7907.

(36) Pal, J.; Manna, S.; Mondal, A.; Das, S.; Adarsh, K. V.; Nag, A. Colloidal Synthesis and Photophysics of $\mathrm{M}_{3} \mathrm{Sb}_{2} \mathrm{I}_{9}(\mathrm{M}=\mathrm{Cs}$ and $\mathrm{Rb})$ Nanocrystals: Lead-Free Perovskites. Angew. Chem., Int. Ed. 2017, 56, 14187-14191.

(37) Zhang, J.; Yang, Y.; Deng, H.; Farooq, U.; Yang, X.; Khan, J.; Tang, J.; Song, H. High Quantum Yield Blue Emission from LeadFree Inorganic Antimony Halide Perovskite Colloidal Quantum Dots. ACS Nano 2017, 11, 9294-9302.

(38) Zhou, L.; Xu, Y.-F.; Chen, B.-X.; Kuang, D.-B.; Su, C.-Y. Synthesis and Photocatalytic Application of Stable Lead-Free $\mathrm{Cs}_{2} \mathrm{AgBiBr}_{6}$ Perovskite Nanocrystals. Small 2018, 14, 1703762.

(39) Creutz, S. E.; Crites, E. N.; De Siena, M. C.; Gamelin, D. R. Colloidal Nanocrystals of Lead-Free Double-Perovskite (Elpasolite) Semiconductors: Synthesis and Anion Exchange to Access New Materials. Nano Lett. 2018, 18, 1118-1123.

(40) Cadmium-Free Quantum Dot Pixelated Color Conversion Layers for the Use in Low Energy Displays. https://www.azonano. com/article.aspx?ArticleID=4356 (accessed October 7, 2019).

(41) Cadmium-Free Quantum Dot LCD Backlight Films for Medium- and Low-Priced LCD Displays. https://www.azonano. com/article.aspx?ArticleID=4168 (October 7, 2019).

(42) Applications of Quantum Dots in Displays. https://www. azonano.com/article.aspx?ArticleID=4828 (October 7, 2019).

(43) Maes, J.; Balcaen, L.; Drijvers, E.; Zhao, Q.; De Roo, J.; Vantomme, A.; Vanhaecke, F.; Geiregat, P.; Hens, Z. Light Absorption Coefficient of $\mathrm{CsPbBr}_{3}$ Perovskite Nanocrystals. J. Phys. Chem. Lett. 2018, 9, 3093-3097.
(44) Talapin, D. V.; Gaponik, N.; Borchert, H.; Rogach, A. L.; Haase, M.; Weller, H. Etching of Colloidal InP Nanocrystals with Fluorides: Photochemical Nature of the Process Resulting in High Photoluminescence Efficiency. J. Phys. Chem. B 2002, 106, 1265912663.

(45) Chen, H.; He, J.; Wu, S. Recent Advances on Quantum-DotEnhanced Liquid-Crystal Displays. IEEE J. Sel. Top. Quantum Electron. 2017, 23, 1-11.

(46) Kim, Y.; Yassitepe, E.; Voznyy, O.; Comin, R.; Walters, G.; Gong, X.; Kanjanaboos, P.; Nogueira, A. F.; Sargent, E. H. Efficient Luminescence from Perovskite Quantum Dot Solids. ACS Appl. Mater. Interfaces 2015, 7, 25007-25013.

(47) Huang, H.; Chen, B.; Wang, Z.; Hung, T. F.; Susha, A. S.; Zhong, H.; Rogach, A. L. Water Resistant $\mathrm{CsPbX}_{3}$ Nanocrystals Coated with Polyhedral Oligomeric Silsesquioxane and Their Use as Solid State Luminophores in All-Perovskite White Light-Emitting Devices. Chem. Sci. 2016, 7, 5699-5703.

(48) Guo, Y.; Shoyama, K.; Sato, W.; Nakamura, E. Polymer Stabilization of Lead(II) Perovskite Cubic Nanocrystals for Semitransparent Solar Cells. Adv. Energy Mater. 2016, 6, 1502317.

(49) Palazon, F.; Akkerman, Q. A.; Prato, M.; Manna, L. X-Ray Lithography on Perovskite Nanocrystals Films: From Patterning with Anion-Exchange Reactions to Enhanced Stability in Air and Water. ACS Nano 2016, 10, 1224-1230.

(50) Meyns, M.; Perálvarez, M.; Heuer-Jungemann, A.; Hertog, W.; Ibáñez, M.; Nafria, R.; Genç, A.; Arbiol, J.; Kovalenko, M. V.; Carreras, J.; Cabot, A.; Kanaras, A. G. Polymer-Enhanced Stability of Inorganic Perovskite Nanocrystals and Their Application in Color Conversion LEDs. ACS Appl. Mater. Interfaces 2016, 8, 19579-19586.

(51) Huang, S.; Li, Z.; Kong, L.; Zhu, N.; Shan, A.; Li, L. Enhancing the Stability of $\mathrm{CH}_{3} \mathrm{NH}_{3} \mathrm{PbBr}_{3}$ Quantum Dots by Embedding in Silica Spheres Derived from Tetramethyl Orthosilicate in "Waterless" Toluene. J. Am. Chem. Soc. 2016, 138, 5749-5752.

(52) Raja, S. N.; Bekenstein, Y.; Koc, M. A.; Fischer, S.; Zhang, D.; Lin, L.; Ritchie, R. O.; Yang, P.; Alivisatos, A. P. Encapsulation of Perovskite Nanocrystals into Macroscale Polymer Matrices: Enhanced Stability and Polarization. ACS Appl. Mater. Interfaces 2016, 8, 35523-35533.

(53) Ling, Y.; Tan, L.; Wang, X.; Zhou, Y.; Xin, Y.; Ma, B.; Hanson, K.; Gao, H. Composite Perovskites of Cesium Lead Bromide for Optimized Photoluminescence. J. Phys. Chem. Lett. 2017, 8, 32663271.

(54) Gomez, L.; de Weerd, C.; Hueso, J. L.; Gregorkiewicz, T. Color-Stable Water-Dispersed Cesium Lead Halide Perovskite Nanocrystals. Nanoscale 2017, 9, 631-636.

(55) Ai, B.; Liu, C.; Wang, J.; Xie, J.; Han, J.; Zhao, X. Precipitation and Optical Properties of $\mathrm{CsPBr}_{3}$ Quantum Dots in Phosphate Glasses. J. Am. Ceram. Soc. 2016, 99, 2875-2877.

(56) Di, X.; Hu, Z.; Jiang, J.; He, M.; Zhou, L.; Xiang, W.; Liang, X. Use of Long-Term Stable $\mathrm{CsPbBr}_{3}$ Perovskite Quantum Dots in Phospho-Silicate Glass for Highly Efficient White LEDs. Chem. Commun. 2017, 53, 11068-11071.

(57) Sun, J.-Y.; Rabouw, F. T.; Yang, X.-F.; Huang, X.-Y.; Jing, X.-P.; Ye, S.; Zhang, Q.-Y. Facile Two-Step Synthesis of All-Inorganic Perovskite $\mathrm{CsPbX}_{3}(\mathrm{X}=\mathrm{Cl}, \mathrm{Br}$, and I) Zeolite-Y Composite Phosphors for Potential Backlight Display Application. Adv. Funct. Mater. 2017, 27, 1704371.

(58) Liu, S.; Luo, Y.; He, M.; Liang, X.; Xiang, W. Novel CsPbI QDs Glass with Chemical Stability and Optical Properties. J. Eur. Ceram. Soc. 2018, 38, 1998-2004.

(59) Lou, S.; Xuan, T.; Yu, C.; Cao, M.; Xia, C.; Wang, J.; Li, H. Nanocomposites of $\mathrm{CsPbBr}_{3}$ Perovskite Nanocrystals in an Ammonium Bromide Framework with Enhanced Stability. J. Mater. Chem. C 2017, 5, 7431-7435.

(60) Pan, A.; Jurow, M.; Zhao, Y.; Qiu, F.; Liu, Y.; Yang, J.; Urban, J. J.; He, L.; Liu, Y. Templated Self-Assembly of One-Dimensional $\mathrm{CsPbX}_{3}$ Perovskite Nanocrystal Superlattices. Nanoscale 2017, 9, 17688-17693. 
(61) Yang, G.; Fan, Q.; Chen, B.; Zhou, Q.; Zhong, H. Reprecipitation Synthesis of Luminescent $\mathrm{CH}_{3} \mathrm{NH}_{3} \mathrm{PbBr}_{3} / \mathrm{NaNO}_{3}$ Nanocomposites with Enhanced Stability. J. Mater. Chem. C 2016, 4, 11387-11391.

(62) Loiudice, A.; Saris, S.; Oveisi, E.; Alexander, D. T. L.; Buonsanti, R. CsPbBr 3 QD/AlO $/ \mathrm{Al}_{\mathrm{x}}$ Inorganic Nanocomposites with Exceptional Stability in Water, Light, and Heat. Angew. Chem., Int. Ed. 2017, 56, 10696-10701.

(63) Huang, H.; Lin, H.; Kershaw, S. V.; Susha, A. S.; Choy, W. C. H.; Rogach, A. L. Polyhedral Oligomeric Silsesquioxane Enhances the Brightness of Perovskite Nanocrystal-Based Green Light-Emitting Devices. J. Phys. Chem. Lett. 2016, 7, 4398-4404.

(64) Li, Z.; Kong, L.; Huang, S.; Li, L. Highly Luminescent and Ultrastable $\mathrm{CsPbBr}_{3}$ Perovskite Quantum Dots Incorporated into a Silica/Alumina Monolith. Angew. Chem., Int. Ed. 2017, 56, 81348138.

(65) Wu, H.; Zhang, Y.; Lu, M.; Zhang, X.; Sun, C.; Zhang, T.; Colvin, V. L.; Yu, W. Surface Ligand Modification of Cesium Lead Bromide Nanocrystals for Improved Light-Emitting Performance. Nanoscale 2018, 10, 4173-4178.

(66) Kirakosyan, A.; Yun, S.; Yoon, S.-G.; Choi, J. Surface Engineering for Improved Stability of $\mathrm{CH}_{3} \mathrm{NH}_{3} \mathrm{PbBr}_{3}$ Perovskite Nanocrystals. Nanoscale 2018, 10, 1885-1891.

(67) Veldhuis, S. A.; Tay, Y. K. E.; Bruno, A.; Dintakurti, S. S. H.; Bhaumik, S.; Muduli, S. K.; Li, M.; Mathews, N.; Sum, T. C.; Mhaisalkar, S. G. Benzyl Alcohol-Treated $\mathrm{CH}_{3} \mathrm{NH}_{3} \mathrm{PbBr}_{3}$ Nanocrystals Exhibiting High Luminescence, Stability, and Ultralow Amplified Spontaneous Emission Thresholds. Nano Lett. 2017, 17, 7424-7432.

(68) Woo, J. Y.; Kim, Y.; Bae, J.; Kim, T. G.; Kim, J. W.; Lee, D. C.; Jeong, S. Highly Stable Cesium Lead Halide Perovskite Nanocrystals through In Situ Lead Halide Inorganic Passivation. Chem. Mater. 2017, 29, 7088-7092.

(69) Wang, C.; Chesman, A. S. R.; Jasieniak, J. J. Stabilizing the Cubic Perovskite Phase of $\mathrm{CsPbI}_{3}$ Nanocrystals by Using an Alkyl Phosphinic Acid. Chem. Commun. 2017, 53, 232-235.

(70) Pan, J.; Quan, L. N.; Zhao, Y.; Peng, W.; Murali, B.; Sarmah, S. P.; Yuan, M.; Sinatra, L.; Alyami, N. M.; Liu, J.; Yassitepe, E.; Yang, Z.; Voznyy, O.; Comin, R.; Hedhili, M. N.; Mohammed, O. F.; Lu, Z. H.; Kim, D. H.; Sargent, E. H.; Bakr, O. M. Highly Efficient Perovskite-Quantum-Dot Light-Emitting Diodes by Surface Engineering. Adv. Mater. 2016, 28, 8718-8725.

(71) Wang, Y.; Yu, D.; Wang, Z.; Li, X.; Chen, X.; Nalla, V.; Zeng, $\mathrm{H}$.; Sun, H. Solution-Grown $\mathrm{CsPbBr}{ }_{3} / \mathrm{Cs}_{4} \mathrm{PbBr}_{6}$ Perovskite Nanocomposites: Toward Temperature-Insensitive Optical Gain. Small 2017, 13, 1701587.

(72) Krieg, F.; Ochsenbein, S. T.; Yakunin, S.; ten Brinck, S.; Aellen, P.; Süess, A.; Clerc, B.; Guggisberg, D.; Nazarenko, O.; Shynkarenko, Y.; Kumar, S.; Shih, C.-J.; Infante, I.; Kovalenko, M. V. Colloidal $\mathrm{CsPbX}_{3}(\mathrm{X}=\mathrm{Cl}, \mathrm{Br}, \mathrm{I})$ Nanocrystals 2.0: Zwitterionic Capping Ligands for Improved Durability and Stability. ACS Energy Lett. 2018, 3, 641-646.

(73) Xu, L.; Chen, J.; Song, J.; Li, J.; Xue, J.; Dong, Y.; Cai, B.; Shan, Q.; Han, B.; Zeng, H. Double-Protected All-Inorganic Perovskite Nanocrystals by Crystalline Matrix and Silica for Triple-Modal AntiCounterfeiting Codes. ACS Appl. Mater. Interfaces 2017, 9, 2655626564.

(74) Kovalenko, M. V.; Protesescu, L.; Bodnarchuk, M. I. Properties and Potential Optoelectronic Applications of Lead Halide Perovskite Nanocrystals. Science 2017, 358, 745-750.

(75) Wei, Y.; Deng, X.; Xie, Z.; Cai, X.; Liang, S.; Ma, P. a.; Hou, Z.; Cheng, Z.; Lin, J. Enhancing the Stability of Perovskite Quantum Dots by Encapsulation in Crosslinked Polystyrene Beads Via a Swelling-Shrinking Strategy toward Superior Water Resistance. Adv. Funct. Mater. 2017, 27, 1703535.

(76) Quan, L. N.; Quintero-Bermudez, R.; Voznyy, O.; Walters, G.; Jain, A.; Fan, J. Z.; Zheng, X.; Yang, Z.; Sargent, E. H. Highly Emissive Green Perovskite Nanocrystals in a Solid State Crystalline Matrix. Adv. Mater. 2017, 29, 1605945.
(77) Xu, J.; Huang, W.; Li, P.; Onken, D. R.; Dun, C.; Guo, Y.; Ucer, K. B.; Lu, C.; Wang, H.; Geyer, S. M.; Williams, R. T.; Carroll, D. L. Imbedded Nanocrystals of $\mathrm{Cs}_{\mathrm{PbBr}}$ in $\mathrm{Cs}_{4} \mathrm{PbBr}_{6}$ : Kinetics, Enhanced Oscillator Strength, and Application in Light-Emitting Diodes. Adv. Mater. 2017, 29, 1703703.

(78) Guhrenz, C.; Benad, A.; Ziegler, C.; Haubold, D.; Gaponik, N.; Eychmüller, A. Solid-State Anion Exchange Reactions for Color Tuning of $\mathrm{CsPbX}_{3}$ Perovskite Nanocrystals. Chem. Mater. 2016, 28, 9033-9040

(79) Bodnarchuk, M. I.; Boehme, S. C.; ten Brinck, S.; Bernasconi, C.; Shynkarenko, Y.; Krieg, F.; Widmer, R.; Aeschlimann, B.; Günther, D.; Kovalenko, M. V.; Infante, I. Rationalizing and Controlling the Surface Structure and Electronic Passivation of Cesium Lead Halide Nanocrystals. ACS Energy Lett. 2019, 4, 63-74.

(80) Liu, Y.; Li, F.; Liu, Q.; Xia, Z. Synergetic Effect of Postsynthetic Water Treatment on the Enhanced Photoluminescence and Stability of $\mathrm{CsPbX}_{3}(\mathrm{X}=\mathrm{Cl}, \mathrm{Br}, \mathrm{I})$ Perovskite Nanocrystals. Chem. Mater. 2018, 30, 6922-6929.

(81) Li, F.; Liu, Y.; Wang, H.; Zhan, Q.; Liu, Q.; Xia, Z. Postsynthetic Surface Trap Removal of $\mathrm{CsPbX}_{3}(\mathrm{X}=\mathrm{Cl}, \mathrm{Br}$, or I) Quantum Dots Via a $\mathrm{ZnX}_{2} /$ Hexane Solution toward an Enhanced Luminescence Quantum Yield. Chem. Mater. 2018, 30, 8546-8554.

(82) Malgras, V.; Tominaka, S.; Ryan, J. W.; Henzie, J.; Takei, T.; Ohara, K.; Yamauchi, Y. Observation of Quantum Confinement in Monodisperse Methylammonium Lead Halide Perovskite Nanocrystals Embedded in Mesoporous Silica. J. Am. Chem. Soc. 2016, 138, 13874-13881.

(83) Demchyshyn, S.; Roemer, J. M.; Groiß, H.; Heilbrunner, H.; Ulbricht, C.; Apaydin, D.; Böhm, A.; Rütt, U.; Bertram, F.; Hesser, G.; Scharber, M. C.; Sariciftci, N. S.; Nickel, B.; Bauer, S.; Głowacki, E. D.; Kaltenbrunner, M. Confining Metal-Halide Perovskites in Nanoporous Thin Films. Science Advances 2017, 3, No. e1700738.

(84) Di, X.; Shen, L.; Jiang, J.; He, M.; Cheng, Y.; Zhou, L.; Liang, X.; Xiang, W. Efficient White LEDs with Bright Green-Emitting $\mathrm{Cs} \mathrm{PbBr}_{3}$ Perovskite Nanocrystal in Mesoporous Silica Nanoparticles. J. Alloys Compd. 2017, 729, 526-532.

(85) Ashley, M. J.; O’Brien, M. N.; Hedderick, K. R.; Mason, J. A.; Ross, M. B.; Mirkin, C. A. Templated Synthesis of Uniform Perovskite Nanowire Arrays. J. Am. Chem. Soc. 2016, 138, 10096-10099.

(86) Abe, S.; Joos, J. J.; Martin, L. I. D. J.; Hens, Z.; Smet, P. F. Hybrid Remote Quantum Dot/Powder Phosphor Designs for Display Backlights. Light: Sci. Appl. 2017, 6, No. e16271.

(87) Brennan, M. C.; Herr, J. E.; Nguyen-Beck, T. S.; Zinna, J.; Draguta, S.; Rouvimov, S.; Parkhill, J.; Kuno, M. Origin of the SizeDependent Stokes Shift in $\mathrm{CsPbBr}_{3}$ Perovskite Nanocrystals. J. Am. Chem. Soc. 2017, 139, 12201-12208.

(88) Sadasivan, S.; Linton, J. R.; Gildea, D. R.; Coe-Sullivan, S.; Shah, S.; Nick, R. J. Quantum Dot Based Lighting. Patent US20120113671A1, 2011.

(89) Sung, J.; Choi, M.; Shin, H.; Jang, D. Method for Preparing Quantum Dot-Polymer Complex, Quantum Dot-Polymer Complex, Light Conversion Film, Backlight Unit and Display Device Having the Same. Patent US20160161066A1, 2014.

(90) Cheon, K.-O.; Gillies, J.; Socha, D.; Duncan, D.; LoCasio, M. Solid State Lighting Devices Comprising Quantum Dots. US8941293B2, 2011.

(91) Werner, M.; Gresty, N.; Pickett, N.; Chalker, P.; Harris, J.; Naasani, I. Multi-Layer-Coated Quantum Dot Beads. US9443998B2, 2014.

(92) Dang, Z.; Shamsi, J.; Akkerman, Q. A.; Imran, M.; Bertoni, G.; Brescia, R.; Manna, L. Low-Temperature Electron Beam-Induced Transformations of Cesium Lead Halide Perovskite Nanocrystals. ACS Omega 2017, 2, 5660-5665.

(93) Pickett, N. L.; Gresty, N. C.; Hines, M. A. 34-1:Invited Paper: Heavy Metal-Free Quantum Dots Making Inroads for Consumer Applications. Dig. Tech. Pap. - Soc. Inf. Disp. Int. Symp. 2016, 47, 425427.

(94) Murphy, J. E.; Beard, M. C.; Norman, A. G.; Ahrenkiel, S. P.; Johnson, J. C.; Yu, P. R.; Micic, O. I.; Ellingson, R. J.; Nozik, A. J. 
PbTe Colloidal Nanocrystals: Synthesis, Characterization, and Multiple Exciton Generation. J. Am. Chem. Soc. 2006, 128, 32413247.

(95) Seth, S.; Samanta, A. Fluorescent Phase-Pure Zero-Dimensional Perovskite-Related $\mathrm{Cs}_{4} \mathrm{PbBr}_{6}$ Microdisks: Synthesis and SingleParticle Imaging Study. J. Phys. Chem. Lett. 2017, 8, 4461-4467.

(96) Diroll, B. T.; Zhou, H.; Schaller, R. D. Low-Temperature Absorption, Photoluminescence, and Lifetime of $\mathrm{CsPbX}_{3}(\mathrm{X}=\mathrm{Cl}, \mathrm{Br}$, I) Nanocrystals. Adv. Funct. Mater. 2018, 28, 1800945.

(97) Cola, M.; Massarotti, V.; Riccardi, R.; Sinistri, C. Binary Systems Formed by Lead Bromide with ( $\mathrm{Li}, \mathrm{Na}, \mathrm{K}, \mathrm{Rb}, \mathrm{Cs}$ and $\mathrm{Tl}) \mathrm{Br}$ : A Dta and Diffractometric Study. Z. Naturforsch., A: Phys. Sci. 1971, 26, 1328-1332.

(98) Saidaminov, M. I.; Abdelhady, A. L.; Murali, B.; Alarousu, E.; Burlakov, V. M.; Peng, W.; Dursun, I.; Wang, L.; He, Y.; Maculan, G.; Goriely, A.; Wu, T.; Mohammed, O. F.; Bakr, O. M. High-Quality Bulk Hybrid Perovskite Single Crystals within Minutes by Inverse Temperature Crystallization. Nat. Commun. 2015, 6, 7586.

(99) Diroll, B. T.; Nedelcu, G.; Kovalenko, M. V.; Schaller, R. D. High-Temperature Photoluminescence of $\mathrm{CsPbX}_{3}(\mathrm{X}=\mathrm{Cl}, \mathrm{Br}, \mathrm{I})$ Nanocrystals. Adv. Funct. Mater. 2017, 27, 1606750. 